\title{
ALGORITMO PARA O RECONHECIMENTO DE CARACTERES MANUSCRITOS
}

Rafael Arthur Rocha Miranda ${ }^{1}$, Francisco Assis da Silva ${ }^{2}$, Mário Augusto Pazoti ${ }^{2}$, Almir Olivette Artero $^{3}$, Marco Antonio Piteri ${ }^{3}$

${ }^{1}$ Discente da Faculdade de Informática da UNOESTE. ${ }^{2}$ Docente da Faculdade de Informática da UNOESTE. ${ }^{3}$ Docente da Faculdade de Ciências e Tecnologia da UNESP

\section{RESUMO}

O reconhecimento de caracteres manuscritos em imagens digitais é uma importante e desafiadora área de estudos da Visão Computacional, com possibilidades de muitas aplicações para facilitar o trabalho do cotidiano das pessoas. Este trabalho apresenta um algoritmo de reconhecimento de caracteres manuscritos com duas propostas de abordagens. A primeira proposta complementa um trabalho anterior de alguns dos autores deste artigo, incluindo 290 novos atributos, baseados em histogramas, Zoning e transformada Hit-or-Miss. A segunda proposta utiliza 79 atributos, obtidos a partir de informações de frequência, distância borda-caractere e densidades, o qual realiza a classificação usando uma abordagem baseada em valores máximos e mínimos de cada atributo para cada tipo de caractere, e uma rede neural Multilayer Perceptron. O grande número de atributos contribui para uma discriminação mais precisa de caracteres, por outro lado, a extração destes descritores é fácil, pois realiza a contagem dos pixels. Assim, o tempo de processamento para a realização dessa tarefa é reduzido. Embora a classificação por meio da rede neural Multilayer Perceptron tenha apresentado uma taxa de acerto superior, o tempo de processamento dos limites máximos e mínimos baseado em classificação é menor, permitindo seu uso em aplicações em situações na qual o tempo de processamento é crítico.

Palavras-Chave: reconhecimento de caracteres; OCR; visão computacional.

\section{ALGORITHM FOR HANDWRITTEN CHARACTER RECOGNITION}

\section{ABSTRACT}

The handwritten character recognition in digital images is an important and challenging area of study in Computer Vision, with several possibilities for applications to facilitate the daily work of the people. This paper presents an algorithm for handwritten character recognition with two proposed approaches. The first proposal complements earlier work by some of the authors of this article, including 290 new attributes, based on histograms, Zoning and transformed Hit-or-Miss. The second proposal uses 79 attributes, obtained from frequency information, distance-edge character and densities, which performs classification using an approach based on maximum and minimum values of each attribute for each character type, and a neural network Multilayer Perceptron. The large number of attributes contributes to a more precise discrimination of characters, on the other hand, the extraction of these descriptors is easy because only performs the pixels counting. Thus, the processing time in this task is reduced. Although the classification using a Multilayer Perceptron neural network achieved a higher hit rate, the processing time of the maximum and minimum limits based classification is smaller, allowing its use in applications where the processing time is critical.

Keywords: character recognition; OCR; computer vision. 


\section{INTRODUÇÃO}

Com as constantes evoluções da imagem digital nos últimos anos, inúmeras possibilidades de aplicações surgiram em grande escala, graças ao alto nível da qualidade das imagens digitais se tornou possível realizar trabalhos (que se considerava impossível, no surgimento das primeiras imagens digitais), como por exemplo, o reconhecimento de caracteres manuscritos em imagens digitais. Devido ao amplo mercado da manipulação de imagens digitais, pesquisas e trabalhos voltados para o reconhecimento de caracteres, tiveram um aumento considerável em relação às décadas passadas. Este assunto apresenta inúmeras aplicações práticas na área de Visão Computacional, e por isso tem sido muito pesquisado. Esta atividade desempenha um importante papel no mundo moderno, podendo ser usado para problemas complexos e realizar alguns trabalhos dos seres humanos. Um exemplo para isso seria um sistema para reconhecimento de código postal de cartas, com o objetivo de ordenálas (CRUZ, 2008; WU, 2003).

O reconhecimento de caracteres manuscritos em imagens digitais é uma importante e desafiadora área de estudos da Visão Computacional (CHAN; YEUNG, 1999; FREITAS, 2001; PARK, 2002; THOMÉ; SILVA,
2003; KAPP, 2004; MONTAÑA, 2007; JAIN; KO, 2008; PEREIRA et al., 2010), e embora essa área tenha apresentado grandes avanços, ainda há muito a ser feito para que uma máquina obtenha um desempenho (no reconhecimento de caracteres manuscritos) parecido ao do ser humano em uma leitura (CRUZ, 2008). A grande dificuldade no reconhecimento se deve ao fato de que os caracteres têm diversas formas de serem representados, tanto os caracteres maiúsculos quanto os caracteres minúsculos, como também os dígitos numéricos e ainda o fato de que diferentes pessoas escrevem de formas diferentes. Devido ao fato da escrita obter formas e estilos diferentes, torna o problema mais complexo para a construção de um sistema OCR (Optical Character Recognition) de alto desempenho.

Alguns algoritmos de reconhecimento de caracteres manuscritos apresentam dificuldades no reconhecimento em algumas situações, ou seja, o algoritmo se "confunde" com as formas de alguns caracteres no momento da classificação, ou seja, o reconhecimento. Essas falhas e dificuldades motivaram a idealização deste trabalho de pesquisa, que tem por objetivo a construção de um algoritmo para o reconhecimento de caracteres manuscritos. 
Apesar de surgir nos últimos anos inúmeras abordagens para solucionar este problema, o processo mais comum envolve as etapas descritas na Figura 1

(MIRANDA et al., 2013).

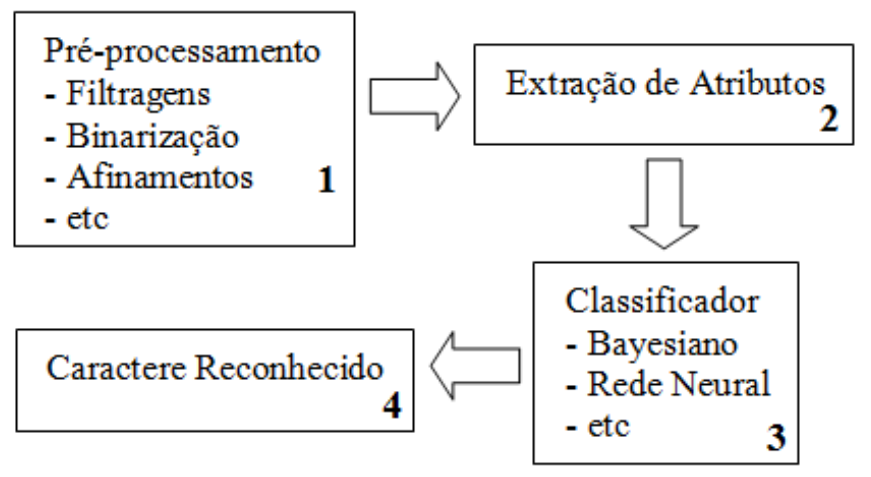

Figura 1. Etapas do processo de reconhecimento de caracteres.

Fonte: (MIRANDA et al., 2013).

$\mathrm{Na}$ primeira etapa são realizadas operações que objetivam uma padronização na aparência dos caracteres, por exemplo, eliminando ruídos, binarizando a imagem e afinando os caracteres. A etapa de extração de atributos é a que merece mais atenção, pois define o sucesso no processo de reconhecimento dos caracteres e, é justamente a etapa que tem demandado mais estudos, pois na literatura há várias técnicas com muitas possibilidades de combinação, as quais podem ser usadas na extração dos atributos. Na etapa 3, o mais comum é usar uma técnica de classificação supervisionada, que busca definir regras que possibilitem predizer a classe a qual pertence cada amostra. Para isso, utilizam-se medições realizadas em caracteres, cujas classes são conhecidas, em um processo de treinamento, para que, em seguida, seja possível reconhecer caracteres com classes desconhecidas, resultando na identificação de caracteres manuscritos.

Um algoritmo com altas taxas de acertos no reconhecimento dos caracteres pode trazer inúmeros benefícios para o dia a dia das pessoas. Alguns exemplos de benefícios são: tradução de prontuários médicos em dados textuais a serem armazenados em bancos de dados, aprimoramento de atividades como o reconhecimento dos valores de cheques bancários, sem a necessidade de uma pessoa digitar os valores (CRUZ, 2008).

Ainda segundo Cruz (2008), há dois tipos de reconhecimento de caracteres, o reconhecimento On-line e o Off-line. No reconhecimento On-line, uma máquina 
sensivel ao toque reconhece a escrita, enquanto o usuário digita, e o reconhecimento é quase instantâneo. No reconhecimento Off-line, os dados são capturados algum tempo após a escrita e, assim, ocorre o processamento e o reconhecimento dos caracteres.

Neste trabalho propõe-se duas estratégias para o reconhecimento de caracteres manuscritos. A primeira estratégia é baseada no algoritmo de Silva et al. (2011), sendo acrescentados novos atributos obtidos com Histogramas, Algoritmo Zoning e Transformada Hit-or-Miss. Na segunda estratégia, adota-se um conjunto de 79 atributos baseados, basicamente, na contagem de pixels de caractere e fundo (matrizes de co-ocorrência, medidas de distâncias borda-caractere e densidades regionais), como alternativa para a descrição dos caracteres. Como esses atributos são baseados em contagens de pixels, o tempo de processamento desta etapa é bastante reduzido. Para a etapa de classificação, além de uma rede neural Multilayer Perceptron, muito usada em diversos trabalhos encontrados na literatura, sugere-se também uma classificação baseada nos valores máximos e mínimos que cada uma das 62 classes de caracteres estudados $(A, \ldots, Z, a, \ldots, Z$ e $0, \ldots, 9)$ assume em cada um dos 79 atributos.
As demais seções deste trabalho estão organizadas da seguinte maneira: na Seção 2 são apresentadas as estratégias propostas neste trabalho para modelar 0 comportamento dos caracteres cujas classes são conhecidas (treinamento) e, então, obter as medidas permitidas em cada classe e usar esta informação na classificação; a Seção 3 apresenta alguns experimentos, os resultados obtidos e a análise de desempenho com a aplicação das duas propostas em dois conjuntos de dados. Por fim, na Seção 4, são apresentados os comentários finais.

\section{DESENVOLVIMENTO DO PROJETO}

O presente trabalho propõe dois tipos de estratégias para o reconhecimento de caracteres manuscritos. A primeira é baseada no algoritmo de Silva et al. (2011; 2012), sendo acrescentados 290 atributos obtidos com Histogramas (Horizontal e Vertical), Algoritmo Zoning e Transformada Hit-orMiss. A segunda estratégia sugere descrever os caracteres de uma forma completamente nova, em que os atributos são obtidos através de informações de frequência, distância borda-caractere e densidades. No processo, os caracteres são segmentados e enquadrados em uma malha com dimensões previamente definidas (70x50) e, em seguida são usados 79 atributos para descrever os 
caracteres manuscritos $(A, \ldots, Z, \quad a, \ldots, z$ e $0, \ldots, 9)$. O uso de tantos atributos se justifica por causa da grande variação de formas e estilos que os caracteres manuscritos podem adotar na sua representação (CRUZ, 2008), e assim, torna-se mais complexo a construção de um algoritmo para o problema proposto.

\subsection{Estratégia baseada em Histogramas} Horizontal e Vertical, Algoritmo Zoning e Transformada Hit-or-Miss

Nesta proposta, foram adicionados novos descritores ao algoritmo de Silva et al. (2011) na tentativa de melhorar a precisão do reconhecimento quando aplicado em imagens contendo caracteres manuscritos.

O algoritmo de Silva et al. (2011), sugere descrever os caracteres, enquadrando-os em uma malha com dimensões definidas previamente e observar as transições entre os níveis de cinza (0 e 1 imagens binárias) dos pixels adjacentes. Assim, uma imagem com dimensões $m \times n$ gera um vetor de transições (atributos) com $m \times n$ posições. Deste modo, o comportamento da sequência dos pixels pode ser determinado e este conhecimento pode ser usado para classificação dos caracteres.

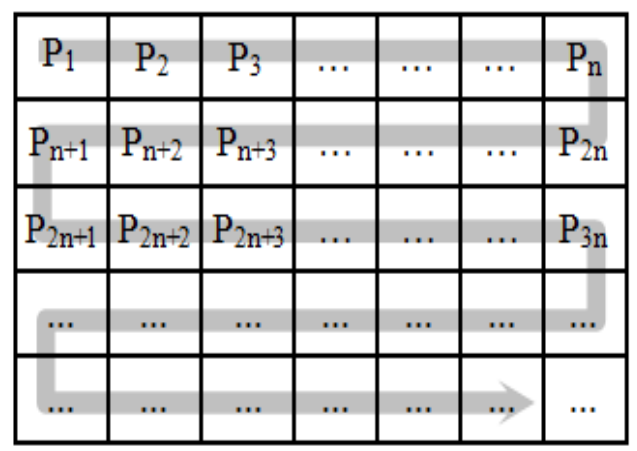

Figura 2. Sequência de pixels na imagem usada.

Fonte: (SILVA et al., 2011).

Uma lista é construída com as transições permitidas para cada caractere, anotando as transições entre os atributos adjacentes em cada uma das classes. Como as imagens utilizadas são binárias, as transições possíveis entre dois pixels são: $\underline{00}$, $\underline{01}, 10$ e 11 . Desta forma, para cada classe são determinadas todas as transições que não ocorrem e a partir dessa informação, os caracteres a serem reconhecidos são incluídos na classe que apresenta o menor número de inconsistência, ou seja, o menor número de transição que não ocorre. A Tabela 1 apresenta as restrições do conjunto de dados. 
Tabela 1. Transições que não ocorrem entre os atributos adjacentes do conjunto de dados.

\begin{tabular}{|c|c|c|c|c|c|c|c|c|c|c|c|c|}
\hline & \multicolumn{12}{|c|}{ Atributos adjacentes } \\
\hline & $a_{11-a_{2}}$ & $a_{2}-a_{3}$ & аз-а4 & a4-a5 & a5-a6 & a6-a7 & $a 7-a s$ & ав-ая & a9-a10 & $a_{10}-a_{11}$ & $a_{11}-a_{12}$ & $a_{12}-a_{13}$ \\
\hline classe 1 & $\frac{\frac{00}{01}}{\underline{11}}$ & $\frac{10}{11}$ & $\frac{00}{10}$ & $\frac{00}{\frac{00}{11}}$ & $\frac{10}{11}$ & $\frac{01}{11}$ & $\frac{\frac{00}{10}}{\underline{11}}$ & $\frac{\underline{00}}{\frac{01}{10}}$ & $\underline{00}$ & $\underline{00} \underline{10}$ & $\begin{array}{l}\frac{00}{01} \\
\underline{10}\end{array}$ & $\frac{00}{\frac{01}{11}}$ \\
\hline classe 2 & $\underline{00} \underline{10}$ & $\frac{00}{\frac{01}{11}}$ & $\frac{00}{\frac{00}{11}}$ & $\frac{00}{\frac{01}{10}}$ & $\frac{00}{\frac{01}{11}}$ & $\frac{10}{11}$ & $\frac{01}{11}$ & $\frac{01}{\frac{01}{11}}$ & $\frac{00}{10}$ & $\underline{00}$ & $\underline{00} \underline{10}$ & $\underline{00}$ \\
\hline
\end{tabular}

Os atributos que foram adicionados ao algoritmo de Silva et al. (2011) foram extraídos a partir do Histograma Horizontal e Vertical, do algoritmo Zoning, e da transformada Hit-or-Miss. imagem, já o Histograma Vertical é calculado pela soma dos pixels pretos de cada coluna da imagem (CRUZ, 2008). A Figura 3 ilustra os histogramas extraídos a partir de um caractere ' 2 ', em uma imagem 70×50.

\subsubsection{Histograma Horizontal e Vertical}

O Histograma Horizontal é calculado pela soma dos pixels pretos de cada linha da

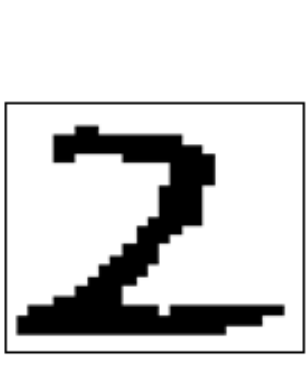

(a)

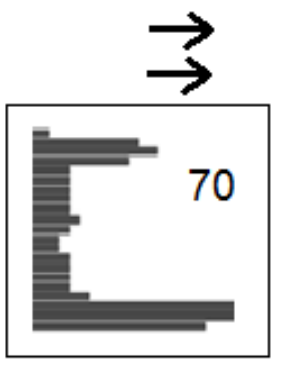

(b)

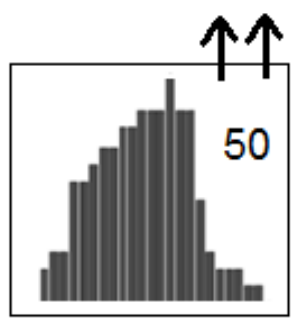

(c)

Figura. 3. a) Imagem Original, b) Histograma Horizontal (70 atributos) e c) Histograma Vertical (50 atributos).

\subsubsection{Algoritmo Zoning}

$\mathrm{O}$ algoritmo de Zoning consiste em dividir uma imagem em zonas, calculando a porcentagem de pixels pretos em cada parte da divisão (CRUZ, 2008). A Figura 4 ilustra um caractere ' 3 ' com divisão $4 \times 4$. 


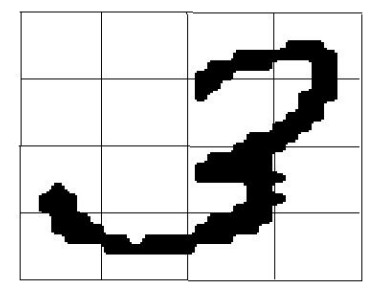

Figura. 4. Divisão $4 \times 4$ para uma imagem de um caractere ' 3 '.

Neste trabalho, foram escolhidas 13 configurações diferentes de divisões $3 \times 1$, $1 \times 3,2 \times 3,3 \times 2,3 \times 3,1 \times 4,4 \times 1,4 \times 4,6 \times 1,1 \times 6$, $6 \times 3,3 \times 6$ e $6 \times 6$. Os resultados de cada configuração foram combinados, formando um vetor contendo 135 características $(3+3$ $+6+6+9+4+4+16+6+6+18+18+36)$.

\subsubsection{Transformada Hit-or-Miss}

A transformada Hit-or-Miss é uma operação morfológica, que pode ser usada para localizar padrões particulares em uma imagem (GONZALEZ; WOODS, 2000). Como acontece com os demais operadores morfológicos que tomam como entrada uma imagem binária e um elemento

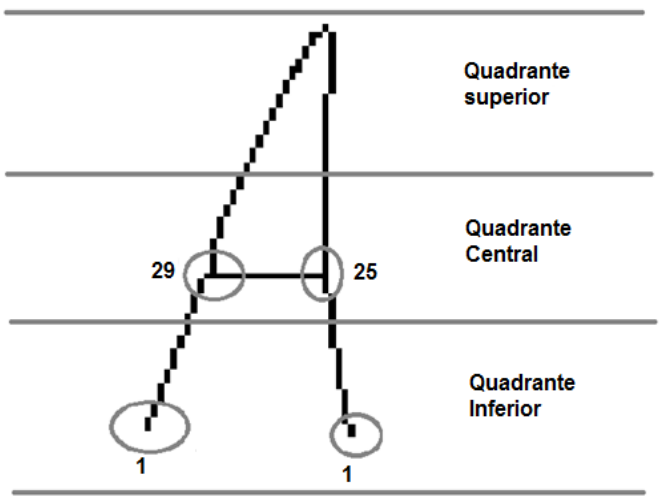

(a) estruturante, e produz outra imagem binária como saída, esta transformada aplica o elemento estruturante a cada ponto da imagem e em seguida compara o elemento estruturante com a matriz da imagem base para a operação.

Foram utilizadas 35 máscaras (elementos estruturantes) para poder extrair os descritores dos caracteres manuscritos a partir das imagens digitais. Para aplicação da transformada Hit-or-Miss, a imagem contendo o caractere manuscrito foi afinada como mostra a Figura 5 (a). Para realizar o afinamento, foi utilizado o algoritmo de Zhang-Suen (GONZALEZ; WOODS, 2000).

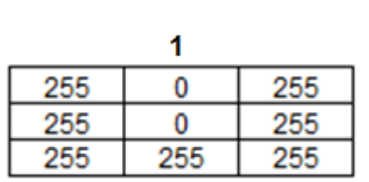

\begin{tabular}{|c|c|c|c|c|}
\multicolumn{1}{c}{29} \\
\hline 255 & 255 & 0 & 255 & 255 \\
\hline 255 & 255 & 0 & 255 & 255 \\
\hline 255 & 255 & 0 & 0 & 0 \\
\hline 255 & 0 & 255 & 255 & 255 \\
\hline 0 & 255 & 255 & 255 & 255 \\
\hline
\end{tabular}

\begin{tabular}{|c|c|c|c|c|}
\multicolumn{5}{|c|}{25} \\
\hline 255 & 255 & 255 & 0 & 255 \\
\hline 0 & 0 & 0 & 0 & 255 \\
\hline 255 & 255 & 255 & 0 & 255 \\
\hline
\end{tabular}

(b)

Figura 5. a) Imagem do caractere ' $A$ ' afinado, b) Pontos terminais e pontos de junções do caractere ' $A$ '. 


\subsection{A Estratégia baseada em informações de} frequência, distância borda-caractere e densidades

Nesta proposta adota-se para cada caractere segmentado e enquadrado em uma malha com dimensões previamente definidas (70x50), um conjunto de 79 atributos baseados, basicamente, na contagem de pixels de caractere e fundo (matrizes de coocorrência, medidas de distâncias bordacaractere e densidades regionais), como alternativa para a descrição dos caracteres. Como esses atributos são baseados em contagens de pixels, o tempo de processamento desta etapa é bastante reduzido. Para a etapa de classificação, além de uma rede neural Multilayer Perceptron, muito usada em diversos trabalhos encontrados na literatura, sugere-se também uma classificação baseada nos valores máximos e mínimos, que cada uma das 62 classes de caracteres estudados $(A, \ldots, Z, a, \ldots, Z$ e $0, \ldots, 9)$ assume em cada um dos 79 atributos.

\subsubsection{Matriz de co-ocorrência}

A Matriz de co-ocorrência geralmente é usada para classificar texturas, ela considera a relação entre dois pixels por vez, um chamado de pixel referência e o outro de pixel vizinho. O pixel vizinho escolhido pode ser vizinho em qualquer direção: por exemplo, para o leste (direita), para o oeste (esquerda), para o norte (acima), para o sul (abaixo), ou na diagonal, para o nordeste, noroeste, sudoeste e sudeste de cada pixel referência (GONZALEZ; WOODS, 2000). Para esta estratégia as matrizes de co-ocorrência são construídas usando os operadores de posição: P1 = "pixel à direita", P2 = "pixel abaixo", P3 = "pixel à direita e acima" e P4 = "pixel à direita e abaixo". Como as imagens utilizadas são binárias, para cada um destes operadores, são obtidas matrizes $2 \times 2$, anotando a frequência em que ocorre as transições entre os pixels vizinhos. As transições possíveis são: 00, 01, 10,11 para cada direção, como ilustra a Figura 7.

\begin{tabular}{|l|l|l|}
\hline & 0 & 1 \\
\hline 0 & $a$ & $b$ \\
\hline 1 & $c$ & $d$ \\
\hline
\end{tabular}

Figura. 7. Matriz de co-ocorrência para imagens binárias com os valores de frequência $a, b, c$ e $d$. 
Desta maneira, cada matriz de coocorrência gera quatro valores (atributos) e, como são usadas quatro matrizes, é gerado um total de 16 atributos com estas matrizes.

\subsubsection{Distância Borda-Caractere}

Os próximos oito atributos são gerados medindo a distância borda-

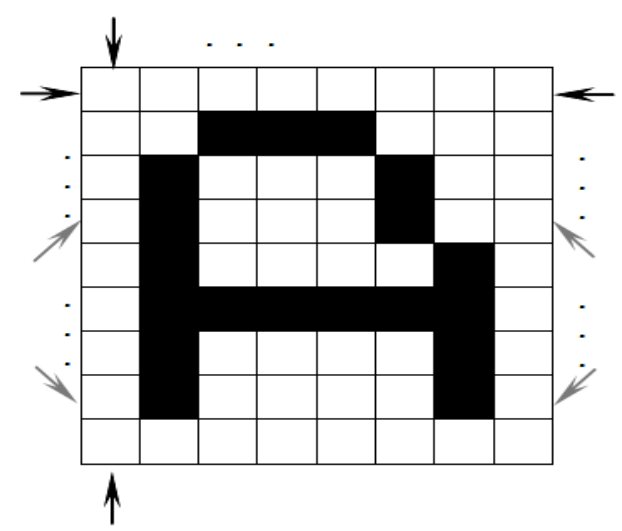

Figura. 8. Oito direções usadas para medir as distâncias borda-caractere.

\subsubsection{Distância borda-caractere com faixa sobreposta}

Os próximos 20 atributos são obtidos aplicando a mesma medida distância bordacaractere, porém, usando faixas sobrepostas, caractere, ou seja, a partir das bordas, são contados os pixels de fundo que antecedem os pixels do caractere nas oito direções $(\rightarrow$, $\leftarrow, \uparrow, \downarrow, \lambda, \forall, \kappa$ e $\boldsymbol{K}$ ) indicadas na Figura 8. (GONZALEZ; WOODS, 2000). 


\subsubsection{Densidade}

Por fim, a imagem 70x50, contendo o caractere, é divida em 35 regiões (10x10) e os últimos 35 atributos são obtidos calculando as densidades de pixels nessas 35 regiões (GONZALEZ; WOODS, 2000). Deste modo, são usados 79 atributos para descrever os caracteres (26 maiúsculos, 26 minúsculos e os 10 dígitos). A Figura 10 ilustra um caractere ' $P$ ' com as densidades por regiões, sendo as regiões mais escuras as de maior densidade.

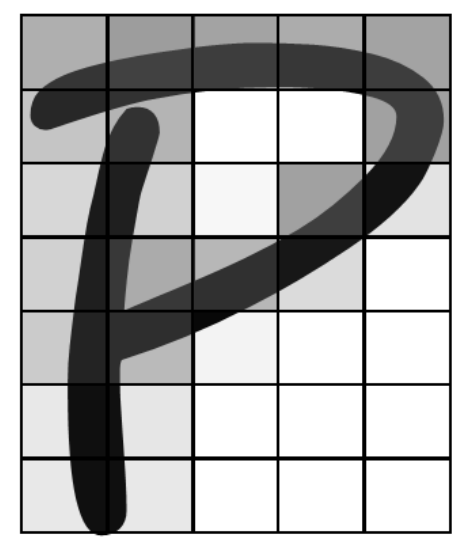

Figura 10. Densidades por regiões. As regiões mais escuras representam uma maior densidade dos pixels.

\subsubsection{Classificação}

$\mathrm{Na}$ etapa de classificação são adotadas duas abordagens. Na primeira, durante o treinamento, são observados os intervalos (máximos e mínimos) que cada classe de caractere apresenta em cada um dos 79 atributos e, em seguida, nas etapas de classificação, os caracteres são colocados nas classes delimitadas por estes valores de máximo e mínimo, obtidos no treinamento. A Figura 11 mostra os máximos e mínimos para o caractere ' $A$ ', 'B', 'C', 'D' e 'E', evidenciando que cada caractere apresenta um certo comportamento para o conjunto de 79 atributos utilizados. 

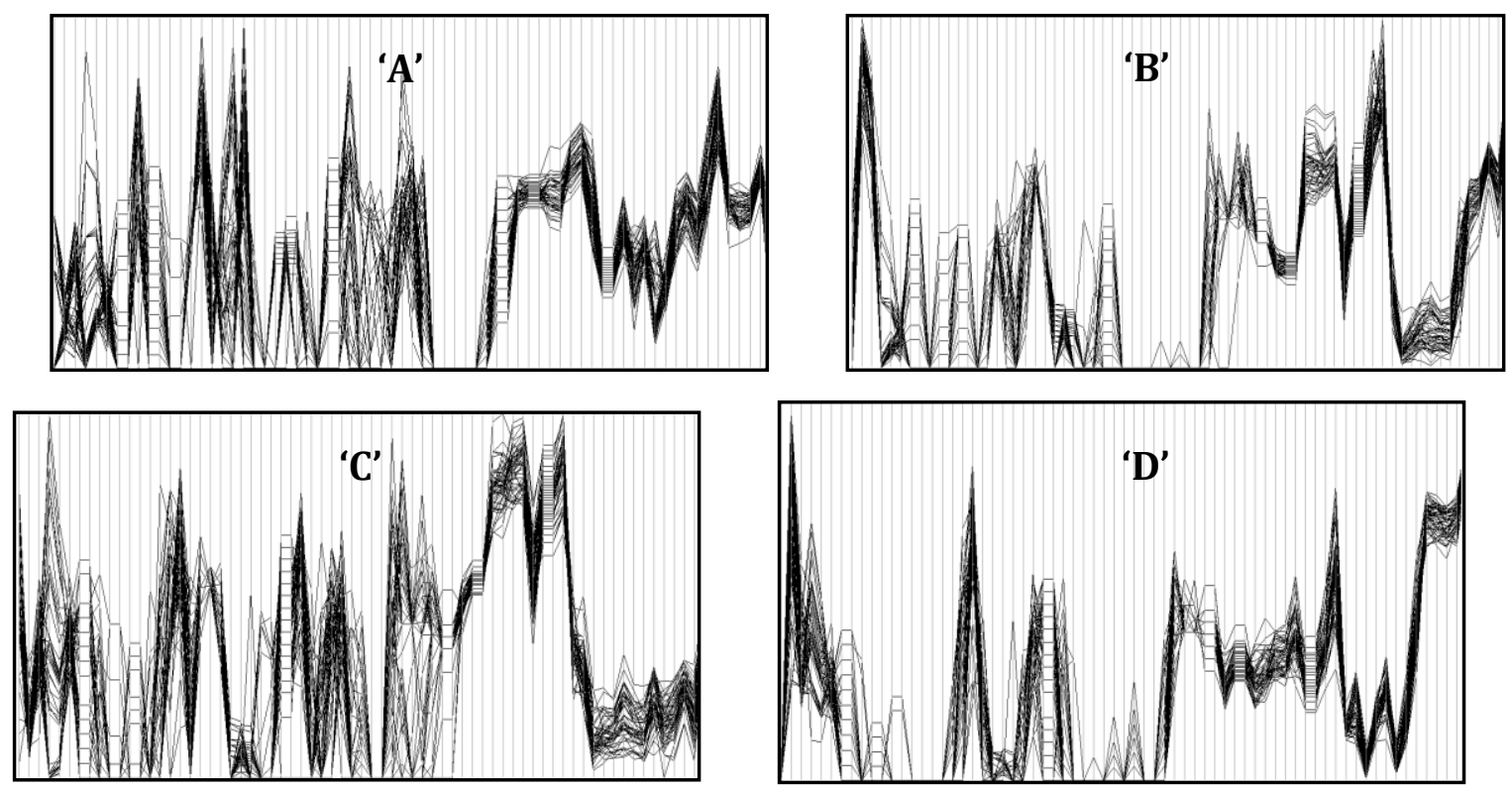

Figura 11. Máximos e mínimos para os 79 atributos dos caracteres ' $A$ ', ' $B$ ', 'C', ' $D$ '.

Além da abordagem dos valores máximos e minímos, a classificação é feita usando uma Rede Neural Multilayer Perceptron, com 79 neurônios na camada de entrada, 70 na camada oculta e 62 na camada de saída, taxa de aprendizagem 0.95 e função de transferência logística.

\section{EXPERIMENTOS}

Nesta seção são apresentados experimentos realizados com as duas estratégias propostas neste trabalho. 0 primeiro conjunto de caracteres usado (caracteres manuscritos) foi obtido com 35 repetições diferentes, feitas por um pessoas, de cada um dos 62 caracteres $(A, \ldots, Z, a, \ldots, z$ e $0, \ldots, 9)$, totalizando 2.170 registros e, na etapa de teste (classificação), foram usadas outras 15 repetições de cada um destes 62 caracteres, totalizando 930 registros (Figura 12).

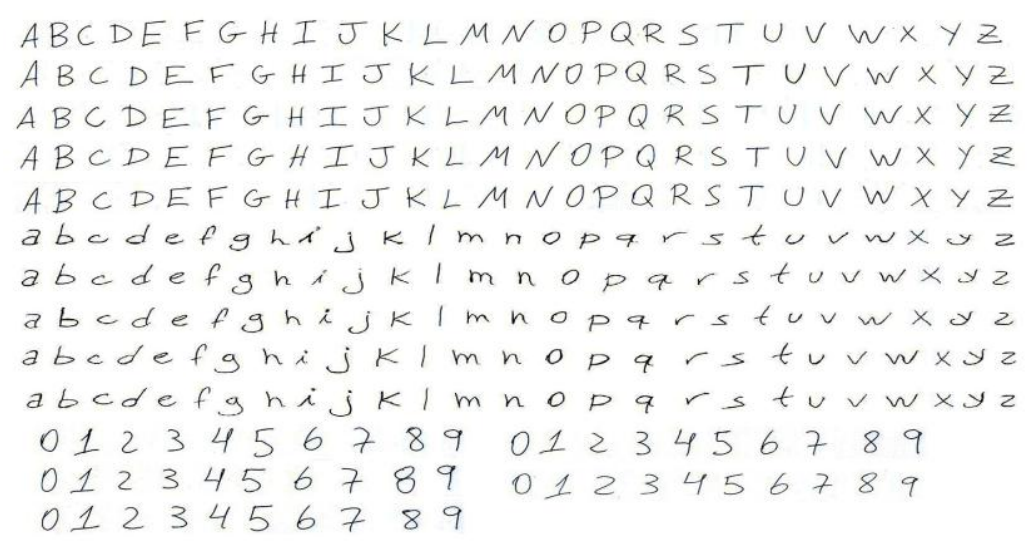

Figura 12. Conjunto de caracteres utilizados. 
O segundo conjunto de caracteres usado é exibido na Figura 13 (caracteres impressos). Trata-se de um conjunto contendo 30 fontes de caracteres em (a), a serem usadas na etapa de treinamento e, 10 fontes de caracteres em (b) (usadas para a etapa de teste).

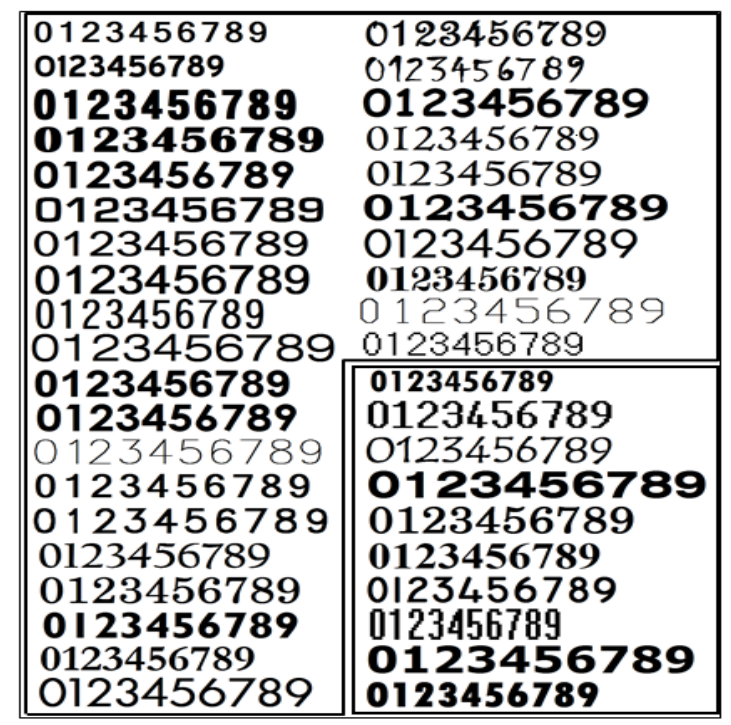

(a)

(b)

Figura 13. Conjunto de caracteres usados no: a) treinamento do classificador (30 fontes diferentes); b) teste (outras 10 fontes diferentes).

\subsection{Experimentos com a estratégia baseada} em Histogramas Horizontal e Vertical, Algoritmo Zoning e Transformada Hitor-Miss

\subsubsection{Experimento I}

Usando a abordagem apresentada por Silva et al. (2011), foi obtida uma taxa de acerto de $66.45 \%$, conseguindo identificar apenas 618 dos 930 caracteres usados no teste (caracteres manuscritos), mesmo usando uma malha razoavelmente densa de
$70 \times 50$ pixels. E utilizando a mesma abordagem de Silva et al. (2011) combinada com as técnicas de Histograma, Zoning, HitOr-Miss, foi obtida uma taxa de acerto de $57,95 \%$, identificando 539 dos 930 caracteres do conjunto de teste representado pela Figura 12, usando a mesma malha de $70 \times 50$ pixels. A Figura 14 mostra os resultados obtidos com esse experimento. 


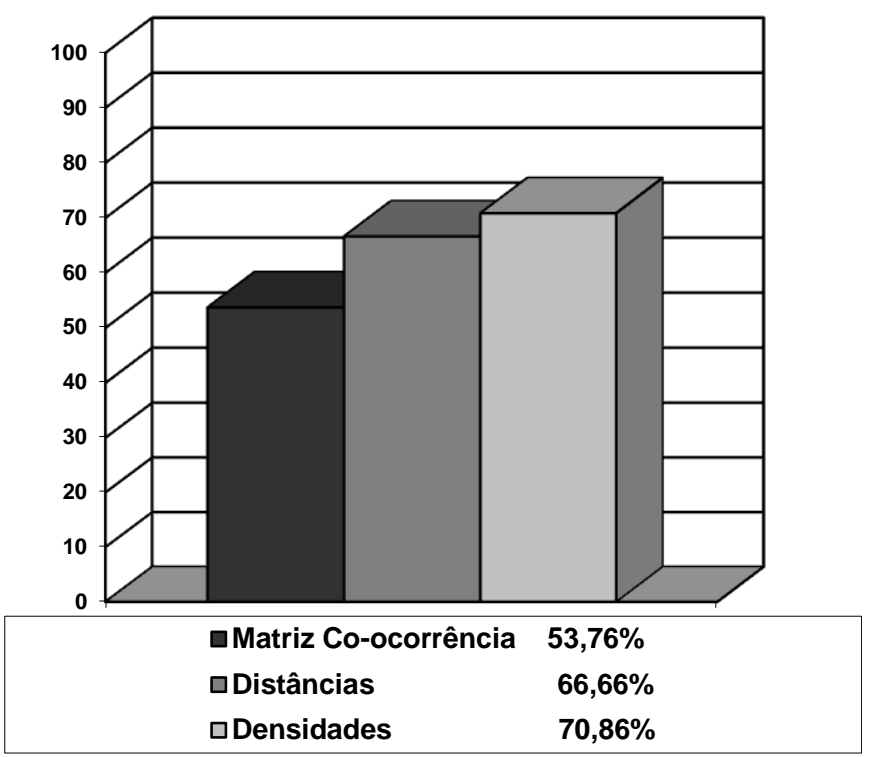

Figura 14. Resultados do Experimento I.

\subsubsection{Experimento II}

Utilizando a abordagem de Silva et al. (2011) combinada com as técnicas de Histograma, Zoning e Hit-Or-Miss, foi obtida uma taxa de acerto de $87 \%$, identificando 87 dos 100 caracteres do conjunto e a somente a abordagem de Silva et al. (2011) obteve 96\%, identificando 96 dos 100 caracteres (caracteres impressos da Figura 13). A Figura 15 mostra os resultados obtidos com esse experimento.

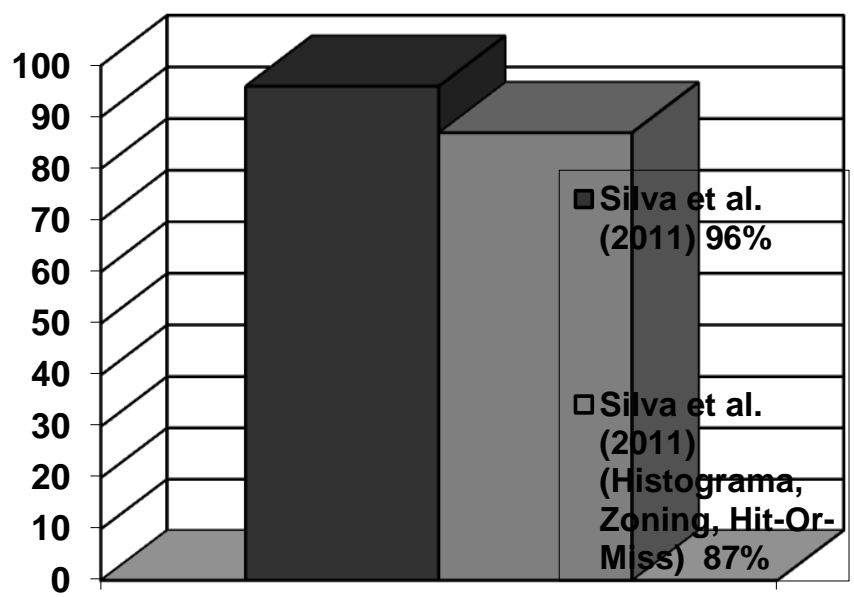

Figura. 15. Resultados do Experimento II (Proposta que não deu certo). 


\subsubsection{Experimento III}

Neste experimento foi utilizado o conjunto apresentado na Figura 12 (caracteres manuscritos) como conjunto de treinamento com 35 repetições e para o teste o conjunto da Figura 16. O resultado foi de $60,34 \%$ identificando 35 dos 58 caracteres para a abordagem de Silva et al. (2011) combinada com as técnicas de Histograma, Zoning e Hit-Or-Miss, e para Silva et al. (2011) foi de $63,79 \%$ identificando 37 do 58 caracteres.

$$
\begin{aligned}
& \text { Esse artigo apresenta um algoritmo } \\
& \text { de reconhecimento de caracteres }
\end{aligned}
$$

a - E888 artigo apresenta Om a6g0rM8m0 a8 p8COnhe Ciment0 a8 ea0acteres

b - 9sse aAAigo apA99eA9a u9 a9AoA9AAo de recAnheci9e9Ao de ca99c9eAes

Figura 16. Conjunto de teste e resultado de reconhecimento para o experimento III

a) Silva et al. (2011). b) Silva et al. (2011) combinado.

Os resultados obtidos em ambas as abordagens utilizadas nesse experimento são mostrados na figura 17.

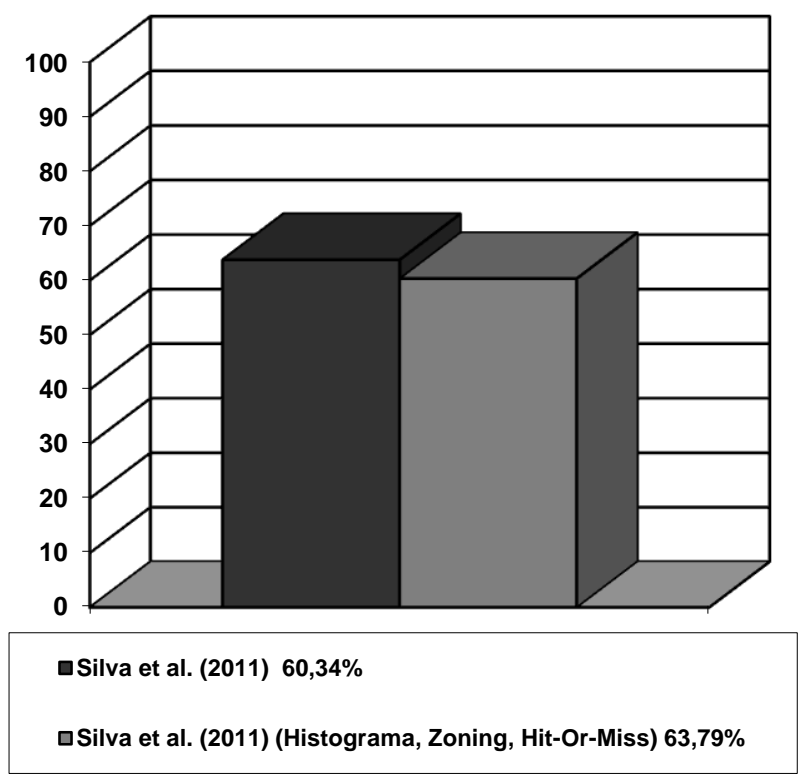

Figura. 17. Resultados do Experimento III (Proposta que não deu certo). 
3.2 Experimentos com a estratégia baseada em informações de frequência, distância borda-caractere e densidades

\subsubsection{Experimento I}

A classificação usando máximos e mínimos obteve um taxa de acerto de $89,46 \%$, identificando corretamente 832 dos 930 caracteres usados no teste. A classificação usando a rede neural Multilayer Perceptron obteve um taxa de acerto de 94,46\%, conseguindo identificar corretamente 880 dos 930 caracteres usados no teste. Usando a abordagem apresentada por Silva et al. (2011), foi obtida uma taxa de acerto de $66.45 \%$, conseguindo identificar apenas 618 dos 930 caracteres usados no teste, mesmo usando uma malha razoavelmente densa de $70 \times 50$ pixels. 0 conjunto utilizado para o treinamento contém 35 repetições e no teste 15 repetições. A Figura 12 Ilustra os conjuntos de caracteres (caracteres manuscritos) e a Figura 18 mostra os resultados.

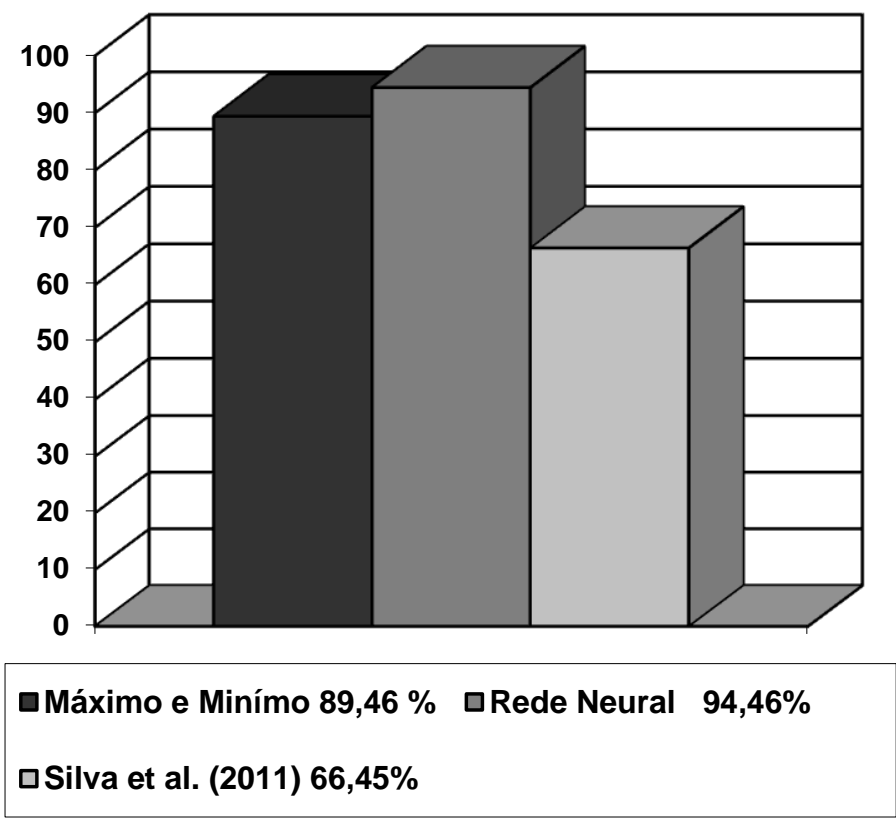

Figura 18. Resultados Experimento I.

\subsubsection{Experimento II}

Utilizando a Figura 14 (a), como conjunto de treinamento, com 30 repetições, totalizando 300 registros e a Figura 14 (b), com fontes diferentes como conjunto de teste, com 10 repetições totalizando 100 registros, obteve-se com a classificação de máximos e mínimos de $94 \%$ de acertos, identificando 94 dos 100 caracteres. Com a rede neural Multilayer Perceptron obteve-se $98 \%$ de acertos, identificando 98 dos 100 caracteres e a abordagem de Silva et al. 
(2011) obteve 96\% de acertos, identificando

os resultados desse experimento.

96 dos 100 caracteres. A Figura 19 apresenta

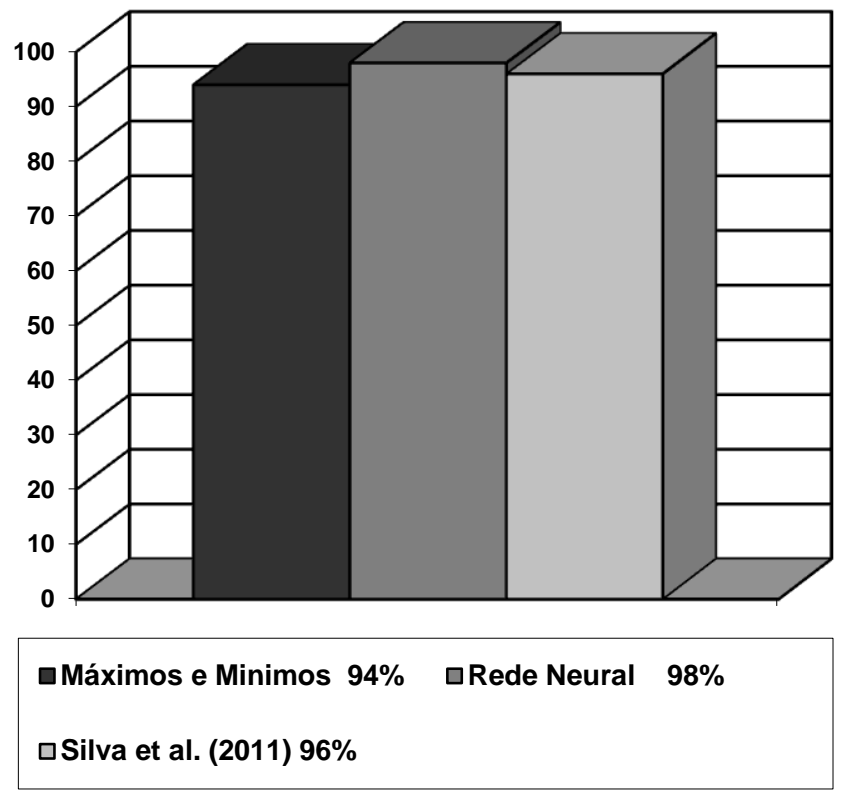

Figura. 19. Resultados Experimento II.

\subsubsection{Experimento III}

com 35 repetições e para o teste o conjunto

Em seguida é apresentado o apresentado pela Figura 20, com 58 experimento realizado com o conjunto de caracteres manuscritos. treinamento apresentado pela Figura 12,

$$
\begin{aligned}
& \text { Esse artigo apresenta um algoritmo } \\
& \text { de reconhecimento de caracteres }
\end{aligned}
$$

\footnotetext{
a - Esse arti9o a6resen9a um al9ori9mo 9e reconnecimen9o 9e carac9eres

b- Bsse artigo apresenta um a7goritmo ae reconnecimento a caracteres

c - E888 artigo apresenta Om a6g0rM8m0 a8 p8COnheCiment0 a 8 eaOacteres
}

Figura 20. Resultado do reconhecimento do experimento III. a) Máximo e Mínimo b) Rede Neural Multilayer Perceptron. c) Silva et al. (2011). 
Usando o método proposto neste artigo, com a classificação baseada em máximos e mínimos, foram reconhecidos 48 dos 58 caracteres (82,75\%). Usando o método proposto neste artigo, com a classificação baseada na rede neural, foram reconhecidos 53 dos 58 caracteres (91,37\%). Usando o método proposto em Silva et al. (2011), foram reconhecidos 37 dos 58 caracteres (63,79\%). A Figura 21 apresenta os resultados desse experimento.

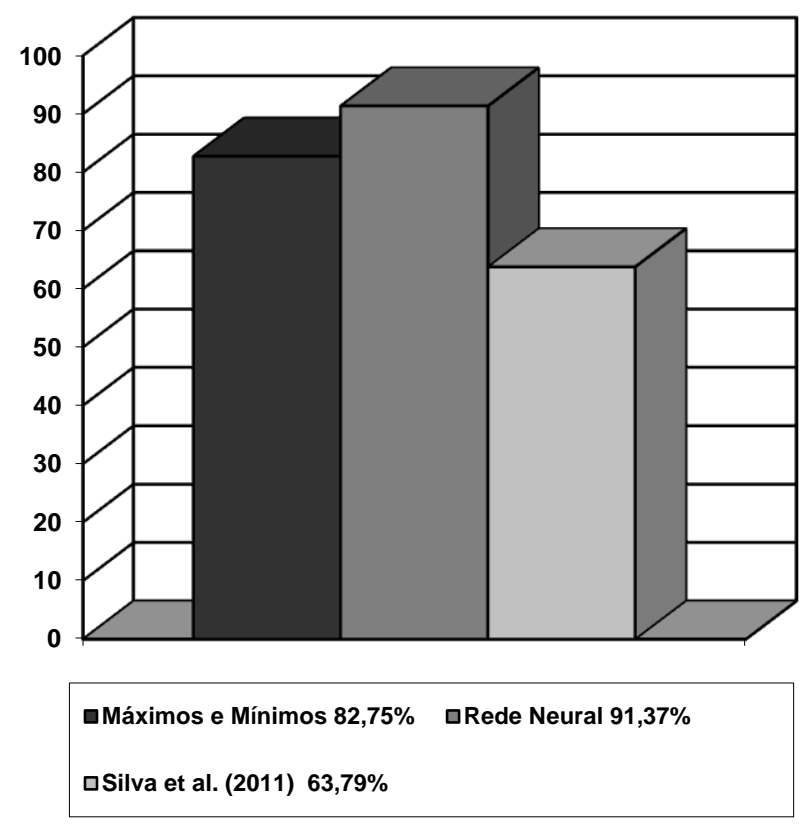

Figura 21. Resultados Experimento III.

\section{CONSIDERAÇÕES FINAIS}

\subsection{Análise de Desempenho}

O tempo médio de processamento para a extração dos 79 atributos dos caracteres ilustrados na Figura 12 é de 0,00678 segundos em uma máquina com um processador Intel Core i5 3317U, com clock de $1.7 \mathrm{GHz}$ e memória de $6 \mathrm{~GB}$. O tempo total de processamento da etapa de treinamento usando a rede neural Multilayer Perceptron foi de 8 minutos e 11 segundos (usando 2.170 registros -35 repetições de 62 tipos de caracteres em 2.000 iterações), enquanto que o tempo de treinamento usando a classificação baseada nos máximos e mínimos foi de apenas 0.98745 segundos, usando o mesmo conjunto de registros. Os tempos médios de reconhecimento por caractere é de 0,0000912 segundos para a classificação usando máximos e mínimos, 0,000161 segundos usando a classificação com a rede neural Multilayer Perceptron e 0,021336 segundos usando a classificação proposta por Silva et al. (2011). 


\section{CONCLUSÕES}

A classificação de caracteres usando as matrizes de co-ocorrência, distâncias borda-caractere e densidades regionais se mostrou bastante eficiente, mesmo com um conjunto de caracteres tão difícil como os caracteres manuscritos, que apresentam uma grande variação, e tem desafiado a maioria dos algoritmos conhecidos. Embora o uso de redes neurais tenha obtido taxas de acerto um pouco superior às obtidas com a classificação baseadas em intervalos de máximos e mínimos, os tempos de processamento desta última abordagem se mostraram mais baixos, indicando a sua aplicação em sistemas de reconhecimento onde o tempo de processamento é crítico, tal como em aplicações de tempo real.

Sugere-se para trabalhos futuros, realizar testes com caracteres manuscritos escritos por outras pessoas e observar os resultados e o comportamento das técnicas de extração de atributos diante de outro conjunto de treino e teste. Além da investigação na literatura sobre outras técnicas de extração de atributos com o intuito de melhorar a taxa de acerto com a classificação baseada em valores máximos e mínimos.

\section{REFERÊNCIAS}

CHAN, K. F.; YEUNG, D. Y. Recognizing on-line handwritten alphanumeric characters through fexible structural matching. Pattern

Recognition, p.1099-1114, 1999. http://dx.doi.org/10.1016/S0031-

3203(98)00155-1

CRUZ, R. M. O. Análise de técnicas de extração de características para 0 reconhecimento de dígitos manuscritos. Recife: Universidade Federal de Pernambuco, 2008.

FREITAS, C. O. A. Uso de modelos escondidos de Markov para reconhecimento de palavras manuscritas. 2001. 188p. Tese (Doutorado) - Pontíficia Universidade Católica do Paraná, Curitiba-PR.

GONZALEZ, R. F; WOODS, R. E. Processamento de imagens digitais. São Paulo: Edgard Blücher, 2000.

JAIN, G.; KO, J. Handwritten digits recognition. Multimedia Systems, Project Report, University of Toronto, Nov. 21, 2008. p. 1-3.

KAPP, M. N. Reconhecimento de palavras manuscritas utilizando redes neurais artificiais. 2004. 98p. Dissertação (Mestrado) - Pontíficia Universidade Católica do Paraná, Curitiba-PR.

MIRANDA, R. A. R. et al. Handwritten character recognition based on frequency, character-edge distances and densities. In: WORKSHOP DE VISÃO COMPUTACIONAL (WVC 2013), 9. Anais... Rio de Janeiro: FGV, 2013.

MONTAÑA, E. G. Digits recognition via neural networks. 2007. Disponível em: http://www.pdf-finder.com/Digitsrecognition-via-neural-networks.html\#.

PARK, J. An adaptative approach to offline handwritten word recognition. IEEE Transactions on Pattern Analysis and Machine Intelligence, v.24, n.7, p. 920-931, 
2002.

http://dx.doi.org/10.1109/TPAMI.2002.1017

619

PEREIRA, J. F. et al. Modular image principal component analysis for handwritten digits recognition. In: INTERNATIONAL CONFERENCE ON SYSTEMS, SIGNALS AND IMAGE PROCESSING, CENTER OF INFORMATICS FEDERAL UNIVERSITY OF PERNAMBUCO RECIFE, BRAZIL, 2010, 17. Anais... Recife: UFPE, 2010.

SILVA, F. A. et al. Um algoritmo rápido para o reconhecimento de caracteres. In: WORKSHOP DE VISÃO COMPUTACIONAL (WVC 2011), 7. Anais... Curitiba: UFPR, 2011. p. 149-154.

SILVA, F. A. et al. Reconhecimento de caracteres baseado em regras de transições entre pixels vizinhos. Avanços em visão computacional. Curitiba: Omnipax, 2012. p. 265-282.

THOMÉ, A. C. G; SILVA, E. Reconhecimento de caracteres manuscritos utilizando time de redes neurais. In: IV Encontro Nacional de Inteligência Artificial, 4. Anais... 2003. p.1316.

WU, M. P. Handwritten character recognition. St Lucia: The School of Information technology and Electrical Engineering the University of Queensland: 2003. 